\title{
Vídeo Síncrono com Preservação Digital e Registro Distribuído como um Serviço para Telessaúde
}

\author{
Denio Mariz Sousa \\ Instituto Federal da Paraíba \\ (IFPB) \\ João Pessoa - PB, Brasil \\ denio@ifpb.edu.br
}

\author{
Guido Lemos Souza \\ Laboratório de Aplicações de \\ Vídeo Digital, UFPB \\ João Pessoa - PB, Brasil \\ guido@lavid.ufpb.br
}

\author{
Cícero I. da Silva \\ Núcleo Telessaúde São Paulo, \\ UNIFESP \\ São Paulo - SP, Brasil \\ cicero.silva@unifesp.br
}

\author{
Giuliano Maia Castro \\ Dynavideo Serviços LTDA \\ João Pessoa - PB, Brasil \\ giuliano@dynavideo.com.br
}

\begin{abstract}
This paper proposes a video as a service (VaaS) platform enabling synchronous video and confidential transmission based in an open and scalable architecture in order to simplify the integration of streaming features in telehealth systems. The platform will provide participant authentication, preservation and recovery of videos with proof of existence, integrity and authenticity through digital certificates and blockchain registration, and the association of the recorded media with the patient's electronic medical record. The goal is that users and health professionals can adopt synchronous video resources to carry out the activities of supervision, teleconsulting, telediagnosis and preceptory of resident doctors with focus on Primary Health Care in remote locations in a safe and reliable way.
\end{abstract}

\section{KEYWORDS}

Telehealth, Telemedicine, Teleconsulting, Digital Health, Blockchain, Digital Preservation

\section{Introdução}

A teleconsultoria é a atividade não presencial realizada entre dois profissionais de saúde para resolução de casos clínicos. O telediagnóstico refere-se à atividade de fazer laudos à distância para exames como um eletrocardiograma ou um Raio X. A teleconsulta é realizada entre profissional de saúde e paciente de forma não presencial. A teleconsulta somente é autorizada pelo Conselho Federal de Psicologia e por Conselhos Regionais de Enfermagem (em poucos casos), sendo ainda proibida na área médica, apesar de grandes avanços recentes em direção a sua regulamentação. Neste trabalho usamos o termo telessaúde para nos referir a uma dessas práticas ou a uma combinação delas.

O Brasil tem concentração geográfica desequilibrada de serviços especializados de saúde (médicos, enfermagem, psicólogos, entre outros), tanto públicos quanto privados. A descentralização de serviços de saúde é uma estratégia para reduzir migração de

In: VI Workshop "O Futuro da Videocolaboração" (WCT-Video 2019), Rio de Janeiro, Brasil. Anais Estendidos do Simpósio Brasileiro de Sistemas Multimídia e Web (WebMedia). Porto Alegre: Sociedade Brasileira de Computação, 2019. (c)2019 SBC - Sociedade Brasileira de Computação. ISSN: 2596-1683 pacientes e melhorar a qualidade do atendimento, mas requer soluções adequadas de telessaúde.

Uma das ferramentas mais eficientes para a telessaúde é o uso de vídeo, seja ele síncrono ou assíncrono. A telessaúde síncrona exige a presença de ambas as partes ao mesmo tempo usando um canal de comunicação que viabilize uma interação em tempo real [1]. A telessaúde assíncrona, caracterizada pela aquisição e armazenamento de dados médicos (como imagens médicas, biossinais, gravações de voz, vídeo etc.) e a transmissão desses dados a um especialista médico em um horário conveniente para avaliação off-line (store-and-forward), não requerendo a presença de ambas as partes ao mesmo tempo.

Os fatores importantes para o avanço do uso da telessaúde, além dos aspectos de disponibilidade de tecnologia e infraestrutura de comunicação, são o suporte legal e a normatização nas áreas de aplicação. Os aspectos relacionados com a tecnologia serão discutidos mais detalhadamente na seção 2 .

Em termos de legislação, o escopo é restrito a cada país individualmente, e cada um avança em ritmo diferente para estabelecer suas regras, que diferem largamente umas das outras em termos do que é permitido.

Quanto à normatização, embora sejam ditadas individualmente por cada país, há avanços de padronização internacional para aspectos como formato de dados, interoperabilidade de sistemas, protocolos de comunicação etc.

O restante deste trabalho está dividido da seguinte forma. A seção 2 apresenta funcionalidades consideradas fundamentais para um sistema de suporte a telessaúde com vídeo. A seção 3 apresenta a proposta para construção de um serviço de "vídeo como um serviço" que incorpora vídeo síncrono, preservação e registro distribuído. A seção 4 apresenta considerações finais com comentários sobre a viabilidade e aplicabilidade da proposta.

\section{Tecnologias e estado da arte em telessaúde}

Em termos de tecnologia, já existem disponíveis atualmente muitos componentes de software e hardware que permitem desenvolver boas soluções de telessaúde. A integração desses componentes para implementação das funcionalidades desejadas é um esforço grande, mas viável, para a construção de uma solução sistêmica eficiente, segura e escalável com foco em telessaúde. 
A seleção das tecnologias a adotar na construção de uma plataforma de serviços para telessaúde depende dos requisitos e características da plataforma de serviços a ser construída.

Algumas características importantes de um sistema de telessaúde podem ser selecionadas com base em iniciativas existentes no mercado ${ }^{1}$, informações de estudos recentes [2], modelos consolidados em outros países [1][3] e também de experiências não comerciais maduras desenvolvidas in-house ou sob encomenda em hospitais privados brasileiros e estrangeiros. Profissionais do Hospital Israelita Albert Einstein, por exemplo, já oferecem serviços em telessaúde desde 2012, prestando assistência a outros centros, hospitais e clínicas nas áreas de UTI, urgência e emergência, teleoncologia e teleneurologia, compartilhando sua expertise e conhecimento em áreas específicas de atuação [4].

Com base nessas soluções, estudos e modelos, listamos a seguir as principais funcionalidades de uma plataforma de serviços de telessaúde, separando-as por categoria. Algumas funcionalidades de uma solução audiovisual de atendimento remoto são igualmente importantes em um bom sistema de teleconferência para qualquer tipo de aplicação, e estão agrupadas na categoria "Funcionalidades Gerais de videoconferência". Outras funcionalidades requeridas para telessaúde que extrapolam as necessidades de uma simples reunião remota, principalmente nos aspectos de segurança e proteção de dados estão agrupadas na categoria "funcionalidades específicas de videoconferência em telessaúde". Nas "funcionalidades adicionais" agrupamos características desejáveis para um sistema de telessaúde, adicionais aos listados nas categorias anteriores. As funcionalidades são listadas a seguir.

\section{Funcionalidades gerais de teleconferência}

- Boa qualidade do vídeo e áudio

- Suporte a dispositivos diversos

- Suporte a variados equipamentos de áudio e vídeo

- Gravação e recuperação de áudio e vídeo

- Videoconferência com uso de navegadores web (flexibilidade)

- Videoconferências autogerenciadas pelo usuário sob demanda

Funcionalidades específicas de teleconferência em telessaúde

- Confidencialidade na transmissão do fluxo de vídeo

- Confidencialidade no armazenamento do vídeo

- Preservação digital de longo prazo

- Autenticação de participantes (profissional e/ou paciente)

- Irrefutabilidade e imutabilidade do vídeo

- Aderência a padrões de interoperabilidade

- Aderência a normas de segurança e privacidade de Registros Eletrônicos de Saúde (RES)

- Política de retenção e descarte de informações

- Gerenciamento de Registros Eletrônicos de Saúde (RES) do paciente

- Suporte a especialidades

${ }^{1}$ ContinuousCare (www.continuouscare.io), Telehealth365

(www.telehealth365.com), Zoom Healthcare (zoom.us/healthcare), VidyoConnect (www.vidyo.com) são alguns exemplos de soluções comerciais bem estabelecidas.
- Suporte a monitoramento de Saúde Domiciliar e Móvel

Funcionalidades adicionais

- Estatísticas e relatórios sobre uso de chamadas, padrões de chamadas, geolocalização dos participantes

- Serviço de Localização de profissionais por especialidade e horário pelo usuário

- Serviço de agendamento de assistência pelo usuário

- Suporte a prontuário e prescrições para o profissional

- Suporte a identidade visual do cliente (clínica, hospital)

- Suporte a especialidades

A maioria dos itens listados acima são autoexplicativos. Teceremos a seguir mais detalhes sobre alguns itens menos comuns.

Os Registros Eletrônicos de Saúde (RES) do paciente são informações médicas do paciente como prontuário, exames, laudos, imagens, prescrições e outras anotações relevantes. Sistemas de telessaúde devem associar o vídeo gravado aos Registros Eletrônicos de Saúde do paciente.

O potencial do Monitoramento de Saúde Domiciliar e Móvel (Home and Mobile Health Monitoring - HMHM) é amplamente reconhecido na política de saúde na Europa e no Reino Unido. Dada a crescente adoção de políticas envolvendo telessaúde, há forte tendência para sua extensão à prestação de cuidados de saúde e de longa duração fora do hospital (em residências) [3]. O suporte a especialidades em um sistema de telessaúde consiste na adição de funcionalidades importantes para algumas especialidades médicas, além daquelas comuns à todas as demais especialidades. Por exemplo, médicos cardiologistas poderão receber dados eletrocardiográficos transmitidos remotamente, em tempo real, para interpretação. Os dados ficam também armazenados no sistema para posterior recuperação.

No aspecto de interoperabilidade, o HL7 (Health Level 7 Data Communications Protocol), por exemplo, é um padrão que orienta a transmissão de informações de saúde e permite a integração de vários dispositivos e aplicações remotos, como aparelhos em UTI, coletores de sinais vitais, exames como eletrocardiograma etc [2]. Nos EUA, sistemas de telessaúde devem ser compatíveis com a HIPAA (Health Insurance Portability and Accountability Act), uma lei que exige eficiência na prestação de serviços de saúde padronizando o intercâmbio de dados eletrônicos e a proteção da confidencialidade e segurança dos dados de saúde através da definição e aplicação de normas. O HIPAA tem regras para:

- Padronização de dados eletrônicos de saúde, administrativos e financeiros do paciente

- Identificadores únicos para indivíduos, empregadores, planos de saúde e provedores de assistência médica

- Padrões de segurança que protegem a confidencialidade e a integridade de "informações de saúde individualmente identificáveis”, passadas, presentes ou futuras.

No Brasil, a Sociedade Brasileira de Informática na Saúde (SBIS) em parceria com o Conselho Federal de Medicina (CFM) 
Video Síncrono com Preservação Digital e

Registro Distribuído como um Serviço para Telessaúde

estabeleceu normas para sistemas de Registro Eletrônico em Saúde, tais como o Nível de Garantia de Segurança 1 e 2 (NGS1, NGS2).

\section{Vídeo como um Serviço para Telessaúde}

Este trabalho propõe o desenvolvimento do V4H - Video Síncrono para Teleconsultoria, Teleconsulta e Telediagnóstico em Telessaúde, um serviço de vídeo síncrono escalável e integrável na nuvem, aplicado às necessidades de teleconsultoria em telessaúde. A ideia é que o V4H, cuja sigla é uma alusão à expressão "video for health", possa ser integrado com facilidade a aplicações de telessaúde existentes ou a serem desenvolvidas, como meio de estender e incorporar funcionalidades complexas de forma transparente e com baixo esforço de integração.

O V4H atuará no modelo Vídeo como um Serviço ("video as a service", VaaS) e disponibilizará recursos de vídeo síncrono multiponto ou ponto-a-ponto em uma rede IP. O serviço incluirá funcionalidades de gerenciamento de chamadas, gestão e autenticação de participantes, captura e processamento de áudio e vídeo, confidencialidade no tráfego dos fluxos de mídia, gravação, autenticação da mídia pelos participantes, registro na blockchain e preservação. O V4H contará com uma interface de programação de aplicações (Application Programming Interface - API) que permitirá que aplicações externas possam ser integradas para fazer uso dos serviços oferecidos por meio da API V4H.

Dessa forma, um sistema de gestão de teleconsultoria, teleconsulta e telediagnóstico em telessaúde que disponha de registro de prontuário eletrônico poderá usar os serviços do $\mathrm{V} 4 \mathrm{H}$ para gerenciar chamadas de vídeo síncrono para teleconsultoria e teleconsulta. Depois da sessão, o V4H fornecerá (via API) um identificador que permitirá ao sistema usuário associar os registros médicos (prontuário e informações) ao vídeo gravado, o qual será preservado pelo tempo requerido pelas normatizações e regulações legais estabelecidas. O sistema de telessaúde sofrerá modificações apenas para integração com a API V4H e não terá necessidade de incorporar as complexas funcionalidades que serão ofertadas pelo $\mathrm{V} 4 \mathrm{H}$, focando na obtenção, guarda e manuseio das informações médicas dos pacientes em si (história, documentos, exames, diagnósticos, plano terapêutico, prontuário etc) de forma segura. Quando o sistema precisar do vídeo ou de outras informações, poderá acessar o V4H para recuperá-los, após devida autenticação.

Uma propriedade muito importante para qualquer sistema de registro de informações no contexto de informações e registros médicos é a "imutabilidade" [5], que é basicamente a capacidade de congelar o registro para que seja permanente, indelével e inalterável, adicionando confiança e integridade para a informação armazenada e compartilhada. De fato, pesquisas recentes [5] [6] [8] apontam tendências de que 1) blockchain se tornará uma parte essencial da gestão de consenso na área da saúde; 2) a integridade dos registros médicos será um caso de uso essencial para blockchain; e 3) veremos uma consolidação substancial da blockchain nas ofertas de cuidados de saúde, o que
Anais Estendidos do WebMedia'2019, Rio de Janeiro, Brasil

valorizará consideravelmente startups com soluções de saúde que usam essa tecnologia. A imutabilidade é um diferencial que será incorporado ao $\mathrm{V} 4 \mathrm{H}$.

O V4H seguirá a legislação pertinente de proteção de dados eventualmente atenderá aos requisitos de segurança como o Nível de Garantia de Segurança 2 (NGS2) [7] e outras normas e padrões de conformidade recomendados. As teleconsultorias síncronas em áudio/vídeo irão trafegar fim-a-fim e serão armazenadas com garantia de confidencialidade (criptografia e protocolos seguros). Haverá mecanismos para garantir a identidade dos usuários ponta-a-ponta, com a possibilidade de assinar a mídia gerada, evitando que um paciente faça uma teleconsultoria com um profissional de saúde desautorizado e que os envolvidos (profissional e, opcionalmente o paciente) possam ser vinculados ao áudio/vídeo gravado (irrefutabilidade).

Esta proposta apresenta vários desafios. Haverá a necessidade de vários serviços coordenados para acomodar o fluxo de trabalho proposto, desde a gerência de usuários, processamento do vídeo síncrono até a preservação da mídia e sua recuperação. A Figura 1 apresenta uma visão de alto nível da arquitetura de serviços do $\mathrm{V} 4 \mathrm{H}$, ainda que de forma preliminar.

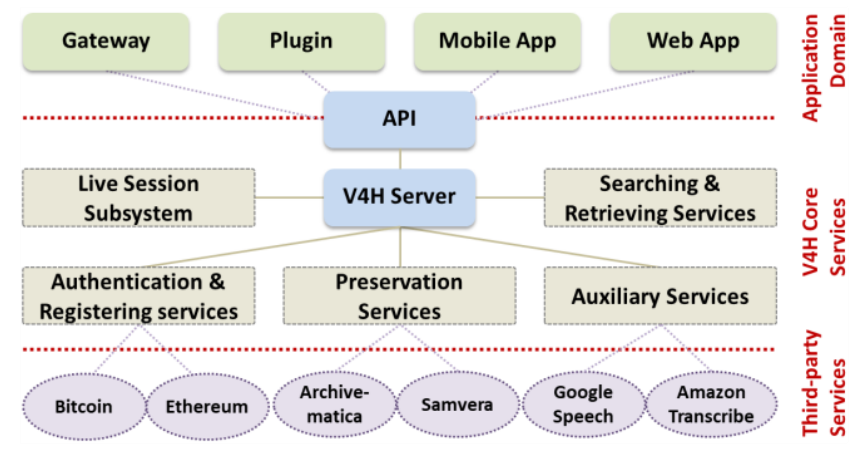

Figura 1 -Arquitetura de serviços do $\mathrm{V} 4 \mathrm{H}$ (visão de alto nível)

No diagrama da Figura 1 o papel de cada componente é o seguinte. O V4H Server é o ponto de entrada do serviço a partir da API, e distribui tarefas para outros subsistemas, serviços e componentes internos. O Live Session Subsystem agrupa componentes com funcionalidades para coordenação dos fluxos de mídia, juntando os vídeos de entrada de cada participantes (ex: médico e paciente) em tempo real, gerando um único fluxo de vídeo de saída a ser preservado, com o efeito picture-in-picture ou a disposição lado-alado. No caso de áudio (sem vídeo), os fluxos de entrada dos participantes serão mixados gerando um único áudio a ser preservado. Também contará com componentes para Live Streaming capaz de receber e gerar fluxos de áudio e vídeo em tempo real durante a sessão para apresentação nos pontos finais envolvidos. A confidencialidade do fluxo deverá ser garantida por componente que decifra/cifra o fluxo ao receber/enviar de/para o cliente final. O cliente final usará componentes (e.g. player) capazes de lidar o fluxo e apresentá-lo na interface do usuário. 
O Authentication \& Registering Services agrupa funcionalidades para calcular e assinar hashes a partir da mídia digital gravada para registro em uma rede DLT (Distributed Ledger Technology), comumente chamada de blockchain. Para tanto, fará uso de um DLT Broker, que decidirá qual a rede blockchain particular usará. Preservation Services é um bloco de serviços responsável pela preservação da mídia gravada e deverá contar com um Preservation Broker, que decidirá qual a plataforma de preservação particular a ser usada. Ambas as funcionalidades de registro em DLT e preservação foram alvo de estudos recentes dos autores na construção de um serviço experimental que demonstrou grande viabilidade para abstrair as plataformas específicas usadas para ambos os tipos de funcionalidade [5].

O Auxiliary Services é um bloco de serviços e componentes que envolve serviços de transcodificação de vídeo para conversão de formatos, auditoria do uso dos recursos e serviços pelos clientes, serviço para registrar, autenticar e definir o papel e as permissões dos usuários no sistema e um Transcription Broker capaz de converter o áudio das consultas em texto usando um serviço de terceiro disponível no mercado. O texto extraído pelo componente de transcrição de áudio poderá ser útil para indexar buscas, tradução para linguagem de sinais (LIBRAS) ou como base de análise e aprendizado para técnicas de IA que auxiliem o diagnóstico, combinando-se com demais informações médicas.

O Searching \& Retrieving Services é um bloco de componentes capazes de a) recuperar ambos a mídia armazenada e o seu registro na blockchain para uma verificação instantânea de integridade, útil para auditoria de imutabilidade; b) recuperar arquivos preservados através do seu identificador; c) gerar fluxo de Video on Demand $(\mathrm{VoD})$ quando requisitado por um player no cliente.

No nível de aplicação, o Gateway exerce o papel de intermediário entre a aplicação de telessaúde usuária/hospedeira e o Serviço $\mathrm{V} 4 \mathrm{H}$, integrando os serviços através de chamadas à API do V4H. Um plugin atua de forma semelhante a um gateway, com a diferença de ser embutido no código executável da aplicação de forma estática ou dinâmica. Mobile App é uma aplicação móvel capaz de acionar serviços através da API do V4H e apresentar adequadamente as informações em uma interface de tablet ou celular. Web App é uma aplicação compatível com tecnologias web/HTML5 capaz de acionar serviços através da API do V4H e apresentar as informações em um navegador.

\section{Considerações Finais}

Muitas soluções existentes no mercado são usadas em telessaúde da mesma forma que são usadas em reuniões de negócio ou outra videocolaboração genérica, sem conformidade com exigências específicas da telessaúde. As poucas soluções existentes que incorporam funcionalidades específicas para videocolaboração em telessaúde (e.g. integração com RES, conformidade com normas e requisitos de segurança) ou não possuem suporte para vídeo síncrono seguro ou são soluções comerciais dispendiosas, provavelmente pela falta de ampla oferta no mercado brasileiro. Soluções com vídeo síncrono para telessaúde com preservação de longo prazo do vídeo digital e garantia de integridade e imutabilidade através de registro distribuído (e.g. blockchain) não foram encontradas na literatura, o que torna esta proposta inovadora do ponto de vista tecnológico. Se considerarmos adicionalmente a possibilidade de oferecer "vídeo como um serviço", tal como aqui proposto, com vídeo síncrono seguro e capacidade de integrar com facilidade a aplicações de telessaúde existentes com custo competitivo, isto seria uma contribuição ao mercado e teria grande aplicabilidade para teleconsultoria, teleconsulta e telediagnóstico em telessaúde.

O público impactado pela disponibilização de um serviço de vídeo síncrono voltado para telessaúde com essas características envolve profissionais de saúde, estudantes e pacientes engajados em serviços de saúde tanto públicos quanto privados.

Os autores desta proposta compartilham a visão de que, no futuro, a telessaúde poderá contar com serviços capazes de integrar informações médicas dos pacientes com outros recursos complementares como imagens, áudio e vídeo de forma confiável e segura para auxiliar a prática profissionais de saúde em sua prática de forma eficiente. Espera-se que os avanços tecnológicos, regulatórios e legais possam permitir a adoção ou ampliação do uso da telessaúde em políticas e programas de saúde pública como ferramenta para redução de custos, otimização de recursos e melhoria da qualidade da atenção à saúde da população em geral.

\section{AGRADECIMENTOS}

A proposta apresentada neste trabalho é parte do projeto selecionado pelo programa de Grupos de Trabalho, edital 2019, com financiamento público pela Rede Nacional de Ensino e Pesquisa (RNP), sob o título "V4H - Video síncrono para teleconsultoria, teleconsulta e telediagnóstico em telessaúde".

\section{REFERÊNCIAS}

[1] Veteran Affairs Telehealth Services. Disponível em https://www.telehealth.va.gov. Acessado em 30-Jul-2019.

[2] Kornak J. (2016) System Requirements for Delivery of Telemedicine Services. In: Cross R., Watson A. (eds) Telemanagement of Inflammatory Bowel Disease. Springer, Cham. https://doi.org/10.1007/978-3-319-22285-1_9

[3] Scottish Centre For Telehealth \& Telecare. A National Service Model for Home and Mobile Health Monitoring. Guidance Release 1.1, 2017. Disponível em https://sctt.org.uk/programmes/home-and-mobile-monitoring/nationalservice-model-home-mobile-health-monitoring. Acessado em 30-Jul-2019.

[4] Telemedicina Einstein oferece mais de 10 serviços de atendimento à distância. Sala de Imprensa da Associação Nacional de Hospitais Privados (ANAHP). Junho/2017. Disponível em https://www.anahp.com.br/noticias/noticiashospitais-membros/telemedicina-einstein-oferece-mais-de-10-servicos-deatendimento-a-distancia. Acessado em 30-Jul-2019.

[5] Tsung-Ting Kuo et al. Blockchain distributed ledger technologies for biomedical and health care applications. Journal of the American Medical Informatics Association, Volume 24, Issue 6, November 2017, Pages 1211-1220. Disponível em https://doi.org/10.1093/jamia/ocx068. Acessado em 26-Mar2019.

[6] Costa, R; Faustino; D; Lemos, G; Queiroga, A; Djohnnatha, C; Alves, F.; Lira, J; Pires, M. Uso Não Financeiro de Blockchain: Um Estudo de Caso Sobre o Registro, Autenticação e Preservação de Documentos Digitais Acadêmicos. Anais do SBRC 2018.

[7] Manual de Certificação para Sistemas de Registro Eletrônico em Saúde. Disponível em http://www.sbis.org.br/certificacao/Manual_Certificacao_SBISCFM_2016_v4-2.pdf. Acessado em 27-Mar-2019.

[8] John D. Halamka et al. Top 10 Blockchain Predictions for the (Near) Future of Healthcare. Blockchain in Healthcare Today ${ }^{\mathrm{TM}}$, ISSN 2573-8240, Volume 2 , 2019. Disponível em https://doi.org/10.30953/bhty.v2.106. Acessado em 26Mar-2019. 